\title{
Restoration of Thymus Function with Bioengineered Thymus Organoids
}

\author{
Asako Tajima ${ }^{1}$ - Isha $\operatorname{Pradhan}^{1} \cdot$ Massimo Trucco ${ }^{1,2,3} \cdot$ Yong Fan ${ }^{1,2,3}$
}

Published online: 1 April 2016

(C) Springer International Publishing AG 2016

\begin{abstract}
The thymus is the primary site for the generation of a diverse repertoire of $\mathrm{T}$ cells that are essential to the efficient function of adaptive immunity. Numerous factors varying from aging, chemotherapy, radiation exposure, virus infection, and inflammation contribute to thymus involution, a phenomenon manifested as loss of thymus cellularity, increased stromal fibrosis, and diminished naïve T cell output. Rejuvenating thymus function is a challenging task since it has limited regenerative capability and we still do not know how to successfully propagate thymic epithelial cells (TECs), the predominant population of the thymic stromal cells making up the thymic microenvironment. Here, we will discuss recent advances in thymus regeneration and the prospects of applying bioengineered artificial thymus organoids in regenerative medicine and solid organ transplantation.
\end{abstract}

This article is part of the Topical Collection on Artificial Tissues

Yong Fan

yfan@wpahs.org

Asako Tajima

ATajima@wpahs.org

Isha Pradhan

ipradhan@wpahs.org

Massimo Trucco

MTrucco1@wpahs.org

1 Institute of Cellular Therapeutics, Allegheny Health Network, 11th Floor, South Tower, 320 East North Avenue, Pittsburgh, PA 15212, USA

2 Department of Biological Sciences, Carnegie Mellon University, Pittsburgh, PA 15213, USA

3 Department of Microbiology and Immunology, Drexel University College of Medicine, Philadelphia, PA 19104, USA
Keywords Thymus $\cdot$ Thymic epithelial cells $\cdot$ Tissue engineering $\cdot$ Thymus organoids $\cdot 3$-D scaffolds $\cdot$ Immune tolerance

\section{Introduction}

In response to selection pressure of evolution, multicellular organisms develop various forms of defense mechanisms to eliminate infectious microbes. Even the most primitive unicellular organisms develop some basic phagocytic ability to neutralize competing microbial agents in the surrounding environment. An effective immune system can recognize a diverse range of pathogens and eliminate them while sparing the organism's own tissues. This, in the vertebrates, is achieved by the coordinated actions of the two arms of the immune systems: the innate immunity and the adaptive immunity. All metazoans (i.e., organisms with three distinct germ layers during early development) develop sophisticated innate immune systems, using germline-encoded innate receptors (commonly referred as pattern recognition receptors, PRRs) to recognize invariant molecular structures that are widely shared by a large group of pathogens, but not produced by the host organisms. These evolutionary conserved targets are called PAMPs for pathogen-associated molecular patterns, presumably representing molecules essential for the survival of the microbes. Such a strategy allows the host organisms to use only a fixed number of germline-inherited PRRs to target diverse populations of microbial pathogens, with low risks of selfdestruction and low "energy expenditure." However, innate immunity is ill equipped to fight against pathogens that are either fast evolving or required specific response. Adaptive system is evolved in Jawed vertebrates, which use somatic mutagenic mechanisms to generate a diverse repertoire of antigen receptors in B and T lymphocytes. In theory, between 
$10^{15}$ and $10^{20}$, possible clonotypes of receptors can be generated through the actions of the recombinant activating genes ( $R A G 1$ and $R A G 2$ ) in human, which enables the immune system to respond to almost all the possible microbial antigens that might be encountered throughout one's lifespan.

As an integral part of the adaptive immune system, the thymus is the primary lymphoid organ responsible for generating a diverse population of $\mathrm{T}$ cells that can recognize and remember the molecular features of invading pathogens [1]. The resulting memory cells can respond promptly to recurrent infections for immune protection. The development of thymocytes with $\alpha \beta$ T cell receptors (TCRs) of high affinity to selfmolecules is effectively undermined within the thymus, where key mechanisms to maintain immunological self-tolerance are taking place. While a lot have been learned over the past decades, the underlying mechanism of thymic "self/non-self" recognition is not fully understood and remains as a major challenge to immunologists.

Most of the solid organs that have a dedicated physiological function(s) vital to the survival of the entire organism (e.g., kidney for filtering blood to remove metabolic waste and excess organic molecules or heart for blood circulation). In contrast, the primary role of the thymus is to promote the differentiation of bone marrow (BM)-derived lymphocyte progenitors (LPs) into mature T cells, which will subsequently exert their physiological function in the periphery. Based on their origin and behavior, cells within the thymus can be categorized into two major compartments: The residential thymic stromal cells (TSCs) which, together with the three-dimensional (3-D) network of extracellular matrix $(\mathrm{ECM})$, constitute the thymic microenvironment and the transient, migratory, and immature thymocytes originated from the BM [2].

After entering the thymus through the blood vessel network at the corticomedullary junction (CMJ) [3, 4], LPs first travel outbound to the subcapsular zone in the "cortex," then moving inward to the "medullary" region, before egressing from the CMJ. Through this journey, LPs follow a well-controlled program of thymopoiesis, differentiating from the CD4-CD8 - double negative (DN) cells, to the CD4+CD8+ double positive (DP) cells, and finally maturing as CD4+CD8- or CD4 -CD8+ single positive (SP) naïve T cells. Cross talk between the developing thymocytes and the thymic stroma, especially the predominant population of thymic epithelial cells (TECs), is essential for the stagewise maturation of thymoctyes.

Three gatekeeping selection events are critical for the generation of the diverse, self-MHC restricted, self-antigen tolerant $\alpha \beta$ TCR repertoire: (1) $\beta$-selection [i.e., the generation of a functional pre-TCR in DN cells, by pairing a germline encoded invariant pre-T $\alpha$ with the TCR $\beta$ chain produced by $\mathrm{V}(\mathrm{D}) \mathrm{J}$ recombination, followed by the TCR $\alpha$ rearrangement and transformation of DN to DP cells], (2) positive selection [the selection of DP cells carrying TCRs that can establish stable TCR and peptide/MHC (pMHC) interactions], and (3) negative selection (the process by which DP and SP cells carrying TCRs with high affinity for self-antigens are eliminated). All these events are dependent on the intimate interactions between the developing thymocytes and TECs in the 3D thymic stromal microenvironment.

Moreover, TECs located at different geometrical regions in the thymic stroma have different functions for T cell development. The subset of TECs in the cortical region (cTECs) is the sole population of TSCs responsible for the positive selection of DP cells. Although the underlying mechanism is not fully understood, recent studies have shown that cTECs are equipped with unique proteasome machineries, which can process antigens differently from other antigen presenting cells (APCs). For example, cTECs express the $\beta 5$ t catalytic subunit of the proteasome, which has different substrate preference from other $\beta 5$ subunits. These properties enable the cTECs to possess and present a unique pMHC ligandome for positive selecting a large variety of functional TCRs [5].

TECs in the thymic medulla (mTECs) are essential for the negative selection of autoreactive $\mathrm{T}$ cells to induce $\mathrm{T}$ cell tolerance [6]. One of the most remarkable features of the mTECs is their capability to ectopically express low levels of antigens that are otherwise restricted to specific tissues and organs. While its underlying mechanisms are not fully understood, two transcription factors have been identified to date to regulate the "promiscuous" expression of a large pool of genes: the autoimmune regulator (Aire) gene and the forebrain expressed zinc finger $2(F e z f 2)$ gene [7••]. Aire was originally isolated as the gene that is mutated in autoimmune polyendocrine syndrome 1 (APS1), a rare, monogenically inherited human autoimmune disease that affects multiple endocrine and exocrine organs [8]. Unlike classical transcription factors that bind to specific DNA sequences for controlling tissue-specific gene expression, Aire binds to non-methylated histone 3 lysine 4 (H3K4) and facilitates the access of the transcriptional factor complexes to otherwise closed chromatin regions in the promoter elements of tissue restricted antigens (TRAs). Airedeficient mice display several similarities with human APS1 [9], indicating the existence of function correlation between thymic TRA expression and the establishment of immune tolerance of peripheral tissues. Indeed, mTEC-specific knockout of Ins 2 , one of the isoforms of the two mouse insulin genes (Ins1 and Ins2) whose thymic expression is regulated by Aire, results in autoimmune destruction of insulin-producing $\beta$ cells in the pancreata of Ins 1-deficient mice. These mice (designated as ID-TEC for insulin-deletion in mTECs) develop full-blown diabetes around 3-4 weeks after birth due to lack of deletion of autoreactive $\mathrm{T}$ cells in the thymus $[10,11]$, highlighting the indispensable role of insulin expression in mTECs in negative selection of insulin-reactive $\mathrm{T}$ cells to establish pancreatic $\beta$-cell immune tolerance.

Notably, although the expression of many thymic TRA is independent of Aire, their ectopic expression in the thymus is 
as important as the Aire-regulated ones in establishing central tolerance of peripheral tissues. For example, islet autoantigen 69 (ICA69), a known $\beta$-cell autoantigen in type 1 diabetes (T1D), is expressed in a wide range of endocrine and exocrine tissues (e.g., the thyroid, the salivary glands, the brain, the stomach, and the testis), in addition to pancreatic $\beta$-cells [12]. ICA69 is expressed in mTECs in an Aire-independent fashion. Mice with mTEC-specific ICA69 deletion spontaneously develop coincident autoimmune responses to the pancreas, the salivary glands, the thyroid, and the stomach, primarily mediated by ICA69-reactive T cells [13]. These results suggest the presence of a direct link between compromised thymic ICA69 expression and autoimmunity against multiple ICA69-expressing organs. Recently, Fezf2 is identified as a transcription factor regulating the mTEC expression of a pool of TRAs that are independent of Aire [7••]. Unlike Aire, which acts to remodel chromatin configuration, Fezf2 directly binds to promoter elements of TRAs to regulate their expression. Mice with TEC-specific deletion of Fezf2 also develop multiple organ autoimmunity. Nevertheless, although the molecular mechanisms of TRA expression in mTECs remain elusive, it is well established that TRA presentation by mTECs is essential for the induction of immune self-tolerance.

\section{The Need to Rejuvenate Thymus Function}

Paradoxically, the thymus itself inherits a transient nature during the organism's lifespan. For reasons largely unknown, thymus precedes other organs in vertebrate animals displaying agerelated senescence. Thymus involution occurs as early as 1 year of age in human. In mouse, the number of TECs peaks at 4 weeks postnatal, before undergoing a persistent decline, in conjunction of gradual increase of thymic fibrosis and adipogenesis [14]. Consequently, the capacity of the thymus to support $\mathrm{T}$ lymphogenesis decreases, resulting in significant decrease of naïve $T$ cell output in middle age and aged individuals. Indeed, the number of thymocytes decreases approximately 30 -fold when mice age from 6 weeks to 24 months [15]. In addition, percentage of recent thymic emigrants (RTEs), the population of naïve T cells that egress the thymus for less than 5 days, drops from 20 to $30 \%$ at 6 weeks to $3 \%$ at 6 months in the spleen, indicating decreased capability of the thymus to generate new T cells to replenish the periphery with age. Similar rapid regression of the thymus cellularity and function is also found in humans beginning as early as 9 months $[16,17]$. Since peripheral Tcell homeostasis is primarily maintained by a balance of newly generated naïve T cells and the homeostatic proliferation of existing cells, diminished thymic output in aged individuals results in numerous changes in the $\mathrm{T}$ cell compartment. These include a decrease in naïve T cell numbers, expansion of small subsets of memory cells, a global shift from naïve to memory phenotype, and a constricted TCR repertoire. Aged T cells also display a number of functional defects, such as reduced proliferation upon mitogen stimulation, altered tonic signaling upon TCR engagement, and reduced IL-2 production $[18,19]$. All these changes contribute to the progressive deterioration of immune function (immunosenescence), resulting in increased susceptibility to opportunistic infections, decreased responsiveness to vaccines, and increased incidence of autoimmunity and cancer.

The thymus gland is also vulnerable to various environmental or pathological insults, which irreversibly compromise its function [20]. In fact, thymic atrophy is a common feature under many acute infectious situations. For example, virus infection (e.g., HIV, rabies virus, measles virus) [21-24] leads to drastic depletion of CD4+CD8+ DP thymocytes. The loss of thymocytes could result either directly from killing by the viruses or indirectly from the deleterious impacts of the elevated proinflammatory cytokines [25]. High levels of glucocorticoid hormones in blood, in response to viral infections, can also trigger massive apoptosis of thymocytes [26]. Other infectious agents (e.g., bacteria, fungus, and helminths) can affect the thymus in a similar fashion. In many cases, infection can also cause severe damage to the TEC compartment, by either inducing apoptosis or accelerating terminal differentiation of TECs. This augmented differentiation results in depletion of the adult TEC progenitor pool and permanent disruption of the thymic stromal microenvironment. Chemotherapies and irradiation treatment of cancers can also cause irreversible damage to the thymic epithelium.

Strategies to rejuvenate thymus function have been primarily focused on treatment with cytokines and growth factors that can promote the regeneration and proliferation of thymic stroma. Aged mice injected with exogenous interleukin 7 (IL7), a vital cytokine for thymocyte development, show increased thymic weight and cellularity [27]. Administration of keratinocyte growth factor (KGF, also known as FGF7) to either aged mice or bone marrow transplanted mice results in increased numbers of early $\mathrm{T}$ lineage progenitors (ETPs) in the thymus and restoration of the thymic architecture. The mode of action of KGF appears to be a favored transient expansion of TEC subsets [28, 29]. Recently, it was found that interleukin 22 (IL-22), an IL-10 gene family member, can act as an effective mediator for TEC regeneration after total body irradiation [30]. Hormone therapies have also been explored to treat age- or injury-related thymus involution. Both growth factors $(\mathrm{GH})$ and ghrelin have been implicated in rejuvenating the thymic stromal microenvironment and improving thymopoiesis [31, 32]. While these approaches are promising for the development of safe and effective clinical therapies for thymus rejuvenation, most of their effects are transient and limited. Systemic administration of these factors can exert unwanted side effects to other tissues/organs. 


\section{Current Status of Thymus Engineering}

Over the years, numerous efforts have been made to use tissue-engineering techniques to develop artificial thymus organoids or grow thymus tissues in vivo as a means to rejuvenate thymus function. One of the major challenges is to recapitulate the unique 3-D architecture of the thymic stroma that is essential for the survival and function of TECs [33, 34]. This is in striking contrast to epithelial cells of other visceral organs, which form a 2-D sheet-like structure on the basement membrane. The expression of genes critical for the specification, proliferation, and function of TECs (e.g., FoxN1, DLL-4, Tbata, MHC II) is dependent on the 3-D configuration of TECs within the thymic stroma [35-39]. When TECs are separated from their thymic microenvironment and placed on irradiated 3T3 cells or other mesenchymal feeders as 2-D culture, most of the TECs start to express markers of terminally differentiated, senescent epithelial cells $[40,41]$ or even transdifferentiate into skin cells [42]. Until recently, the lack of a culture system to properly grow and propagate TECs in vitro has hampered the application of modern gene editing techniques to genetically modified TECs for therapeutics.

Reaggregate thymus organ culture (RTOC) is one successful way to generate 3-D culture of thymus organoids. TSCs are isolated from day 14.5-16.5 mouse embryos, mixed with LPs or developing thymocytes as aggregates and cultured on $0.8-\mu \mathrm{m}$ filters placed on top of dental sponges in petri dish $[43,44]$. Similar approach has been used to culture postnatal human thymic reaggregate units comprised of TECs, isolated thymic mesenchymal cells and CD34+ cord blood progenitors [45•]. In both cases, the microenvironments of these thymic reaggregates can support limited thymopoiesis both in vitro and in vivo. Seeding isolated TECs into matrigel or other collagen-based 3-D metrical systems has also been explored and shown to be able to achieve limited success [46]. Artificial 3-D matrix can also support TEC survival and thymocyte development to certain extent [47]. Moreover, it has been shown recently that supplementing 3-D artificial matrix with dermal fibroblasts derived from the human skin can support the culture of mTECs and retain some of their key molecular features for negative selection (e.g., expression of TRAs) [48].

The major difficulty to reproduce the microenvironment of the thymus is the complexity of its extracellular matrix (ECM) composition and organization. It is now recognized that ECM is not merely a passive support structure but a dynamic system that programs cellular functions through physicochemical means [49-51]. Epithelial cells polarize and migrate by anchoring to fibronectin via integrin receptors [52]. The resulting mechanotransduction alters cellular phenotypes via ERK, Rho, and other signaling pathways. ECM also serves as a reservoir for chemokines, growth factors, and cytokines that make up the unique stromal microenvironment essential for controlling the physiology of a given organ [53]. These ECM properties are fundamental to organ development. Moreover, precise spatiotemporal integration of physicochemical cues is necessary for the colonization, survival, and differentiation of stem/progenitor cells. While significant advances have been made in recent years in the development of both synthetic/ natural biomaterials and 3-D fabricating/printing techniques, it remains difficult to artificially replicate the ECM microenvironments and the complex molecular events within [54].

Using biologic scaffolds prepared from decellularized tissues and organs is an alternative, effective shortcut to reproduce the genuine ECM microenvironment of a particular tissue or organ [55-57]. Numerous physical (e.g., mechanical agitation and snap freezing), chemical (e.g., non-ionic detergents and SDS), and enzymatic (e.g., trypsin and DNase) methods have been developed for induction of cell lysis, removal cellular debris, and renaturation of the protein matrix. With a combination of these approaches, biologic scaffolds have been successfully prepared from various organs and tissues, such as the heart, liver, kidney, pancreas, lung, skeletal muscles, and trachea. Some of these have even been engineered further in bioreactors to support the tissue regeneration from the seeded parenchymal cells, and limited but encouraging function recovery was achieved in preclinical studies [58-64]. Similar technology has already been applied to manufacture and implant relatively simple tissues, such as tissue engineered vascular graft and trachea, in patients with limited success $[65,66]$.

Recently, functional thymus organoids have been successfully constructed by repopulating decellularized thymus scaffolds with TSCs (including TECs, thymic fibroblasts, and endothelial cells) isolated from young adult mice, in conjunction with bone marrow progenitors $[67 \bullet \cdot]$. The microenvironments of the thymus scaffolds can support the survival and function of adult TECs in vitro, without changing their unique molecular properties. When transplanted into $\mathrm{T}$ cell-deficient athymic nude mice, the bioengineered thymus organoid can effectively attract the homing of LPs from the host's bone marrow and supports the generation of a complex T cell repertoire. B cells in the treated mice can undergo affinity maturation and class switching upon immunization with model antigens, indicating assistance from helper T cells. When challenged with allogeneic skin grafts, the treated mice can effectively mobilize cytotoxic $\mathrm{T}$ cells for rapid rejection. In addition, nude mice transplanted with thymus organoids constructed with donor MHC-expressing TECs display donor-specific tolerance to skin grafts but can promptly reject third-party allogeneic skin grafts. This study shows that the bioengineering approach to regenerate thymus can not only generate a functional $\mathrm{T}$ cell compartment but also serve as an immunomodulating tool to modify the identity of "immunological self" and introduce donor-specific immune tolerance $[67 \bullet \bullet]$. 


\section{Major Obstacles to be Circumvented}

While successful to a certain extent in preclinical mouse models, major challenges remain. One of the major caveats is that TECs are isolated and injected into the thymus scaffold as single cells, resulting in loss of cell-cell contact and geometrical organization of TECs in the bioengineered thymus organoids. In contrast, cTECs and mTECs are compartmentalized into distinct geometrical regions in an intact thymus to support specific stages of thymopoiesis. To improve the organization of TEC subsets in the bioengineered thymus, 3-D biofabrication tools to promote the aggregation of TECs can be utilized. Recently, we incorporated TEC-specific antibodies in a polypeptide-based, self-assembling hydrogel system and have successfully generated mini aggregates of TECs [68]. When these 3-D aggregates of TECs were transplanted into athymic nude mice, they could efficiently support Tlymphogenesis. Thus, by either self-assembling or 3-D printing, biocompatible hydrogel systems can serve as versatile tools to further improve the design and organization of the bioengineered thymus organoids.

Another advantage for incorporating biomaterials into thymus bioengineering is that it provides a means to further improve the vasculature of the organoid. Similar to situations in transplantation of solid organs, bioengineered organoids need to be connected to the vascular system to prevent ischemiainduced massive cell death [69]. While the ECM of the vascular framework is well preserved in the decellularized scaffold, significant loss of factors essential for angiogenesis might occur. Previous studies have shown that VEGF produced by the thymic stroma induces robust angiogenesis and is critical for thymic growth in neonatal mice [70, 71]. Moreover, aggregates of human thymic TECs and VEGFexpressing thymic mesenchyme can recruit postnatal human hematopoietic progenitor cells and promote their differentiation into T cells in NSG (NOD scid gamma) humanized mice [45•]. Promoting vascularization with factors essential for angiogenesis embedded in biomaterials could be an effective way to improve the survival and function of the bioengineered thymus organoid engraftment.

\section{Novel Sources of TECs}

Another big challenge facing thymus bioengineering is the limited number of TECs that can be harvested from the adult thymus, together with our incapability to efficiently expand them ex vivo to combat this shortage. For reasons largely unknown, the total numbers of TECs start to decrease at very young age (about 4 weeks postnatal in mouse and 1 year after birth in human), and such declines accelerate at puberty. Recently, a number of studies have shown independently that the numbers of clonogenic units, which presumably represent the proliferative thymic epithelial progenitor cells (TEPCs), drop drastically within the first week after birth $[72,73$, 74•]. How to prevent such early loss of TEPCs and expand them either in vitro or in vivo remains a challenging task.

What hampers the advance of the field is the elusive nature of postnatal TEPCs. On the contrary, much more have been learned regarding the existence and identity of fetal TEPCs, which possess the capability to regenerate the entire TEC compartment. A single mouse TEC isolated at embryonic day 12.5 (E12.5) is able to generate both mTECs and cTECs when injected into fetal thymus lobes, indicating that all TEC subsets have one common origin [75]. Results from fetal tissue transplantation experiments demonstrate that a few placenta-expressed transcript-1 (Plet-1)-expressing E15.5 fetal TECs are sufficient to generate a fully functional thymus in athymic nude mice [76]. Furthermore, when a conditional mutant allele of Foxn1 is converted to a functional wild-type allele in vivo, a single TEC can form a small thymic lobule that is comprised of both cortical and medullary regions and is able to support normal thymopoiesis [77, 78]. These remarkable findings conclusively demonstrate the existence of Foxn1-expressing, fetal TEPCs that can give rise to a complete and functional thymic microenvironment.

However, it is not known how embryonic TEPCs are related to the putative adult TEPCs. To date, the mechanisms underlying TEC homeostasis in postnatal/adult thymus remain largely unclear. To search for the putative progenitors of the adult thymic epithelium, investigators have turned to some of the wellestablished, self-renewal systems (e.g., bone marrow, intestinal stem cell crypts, hair follicles and dermis), and modeled TEC regeneration accordingly [79]. All these systems can effectively maintain long-term homeostasis and are able to promptly regenerate/repair damaged tissues from pathological insults. Two prominent features are common to the stem cells/ progenitors in these systems: (1) their capability to undergo fast division upon stimulation and (2) their capability to stay quiescent and retain labeling of nucleoside analogues (e.g., BrdU) for long-term. These properties not only enable them to quickly replenish the lost cells and maintain tissue homeostasis but also prevent precocious depletion of the stem cell pools.

The labeling-retaining approaches have been used in many studies to identify the subsets of quiescent TECs in postnatal mouse, which are presumably TEPCs [80, 81•, 82]. Using Ki67 and BrdU labeling approach, Gray et al. demonstrated that TECs were highly proliferative at early postnatal stage, to support the rapid expansion of the TEC compartment [83]. The turnover of the TECs is rapid, with an estimation about 10 14 days at 4 weeks but drastically diminishes soon after the initiation of thymic involution. Further experiments showed that after an initial pulse of nucleus label agents at young age, a small fraction of the quiescent label-retaining cells (LRCs) persisted for months in the TEC compartment. Based on the differential expression of common TEC surface markers (e.g., MHC II, 
Ly51, and UEA-1), Wong et al. grouped the adult TECs into different subsets and found that LRCs preferentially reside within the $\mathrm{MHCII}{ }^{\mathrm{lo}} \mathrm{Ly} 51^{+} \mathrm{UEA}^{-1}{ }^{\text {low }}$ subset (designated as $\mathrm{CTEC}^{\mathrm{lo}}$ ) [84 $\bullet$. Interestingly, these cells have high levels of cell-surface expression of both Sca-1 and $\alpha 6$ integrin, a feature shared by stem cell/progenitors in many epithelial tissues, including the epidermis. Furthermore, when mixed with fetal thymus cells in RTOC and transplanted into the athymic nude mice, cTEC $^{\text {lo }}$ cells form small, isolated colonies distributed throughout the thymus grafts, indicating their potential to proliferate and differentiate into various TEC subsets.

While these studies clearly indicate the existence of LRCs in the postnatal thymus, whether they are responsible for maintaining TEC homeostasis is not clear. The postnatal TEC compartment undergoes a chronic decay from early age even under normal physiological conditions, and the thymus gland requires continuous LP input to maintain thymopoiesis and thymic stromal homeostasis. Perinatal loss of thymic tissue is not compensated for in later life, suggesting that the regenerative capacity of the postnatal thymic epithelium is rather limited. In a recent perspective review, Boehm and colleagues suggest that thymus involution is an evolutionarily conserved, natural defense mechanism to prevent the overproduction of T cells [85]. Thus, lessons learned from homeostatic model systems (e.g., hematopoietic stem cells and intestinal stem cells) might not necessarily be translatable to the maintenance of postnatal TEPCs. An alternative, but not mutual exclusive, mechanism is that the size of the thymic microenvironment is primarily maintained by proliferating the existing TECs, like liver and pancreatic islets.

Recent studies have shed light on the molecular mechanisms underlying postnatal TEC proliferation. Mice overexpressing Cyclin D1 under the regulatory control of a keratin 5 (K5) promoter display continuous growth of the thymus and are protected from thymus involution [86]. In contrast, knocking out p63, a member of P53 family of tumor suppressors, in TECs leads to thymus hypoplasia, presumably due to the loss of TEPCs [87, 88]. Foxn1 is another key regulator of adult TEC regeneration. In normal thymus, the expression of Foxn1 is highly heterogeneous, and the numbers of TEC with low levels of Foxn1 increase with age [89]. Mice with increased levels of Foxn1 expression in TECs are resistant from thymic involution [90]. These studies highlight the possibilities of targeting these molecules to expand TEPCs from aged donors.

Alternative sources of TECs for therapeutics have also been actively explored. These include controlled differentiation from embryonic stem cells (hESCs) [91•, 92•, 93], induction of differentiation from induced pluripotent stem cells (iPSCs) [72, 94, 95], or transdifferentiation from mouse embryonic fibroblasts (MEF) [96••, 97]. When these TEC-like cells are transplanted into nude mice or NSG humanized mice, functional $\mathrm{T}$ cells can be generated in vivo. While promising, none of these approaches have been able to so far differentiate the stem cells into a defined population of TEPCs in vitro or in vivo.

\section{The Prospect of Thymus Bioengineering in Therapeutics}

Until today, thymus transplantation is primarily used as an investigational treatment for infants with congenital athymic anomalies, such as "complete" DiGeorge syndrome, a genetic disorder resulting from microdeletion on chromosome 22 at band 22q11.2 (also known as the DGS region), or Foxn1 deficiency [98]. Without intervention, these conditions are fatal. Most of the affected children die before 2 years of age due to opportunistic infections. Typically, donor thymic tissues can be obtained from infants undergoing surgeries for treatment of congenital heart diseases, during which the pediatric cardiac surgeons routinely remove the thymic tissue to gain access to the surgical field. Only thymic tissues obtained from donors under 9 months of age are normally used for transplantation, as the chances for donors to experience viral infections (e.g., cytomegalovirus and human herpes virus) increase significantly with age, which, if passed over to the immune deficient recipients, will be detrimental. In addition, the thymus drastically loses its capability to regenerate 1 year after birth. Successful thymopoiesis is observed in more than $70 \%$ of treated DGS patients, and many of them have been successfully weaned off the immunoglobulin replacement therapy. These encouraging results indicate the clinical feasibility of the protocol and highlight the potential of the thymus transplantation approach in regenerating adaptive immunity. It is conceivable that in the future, with further optimization, patients with thymus agenesis can be transplanted with bioengineered thymus organoids constructed from either cryopreserved donor TECs expanded ex vivo or even TEC-like cells derived from the patient's own cells (e.g., induced from iPSCs or transdifferentiated from dermal fibroblasts).

The long-term immunological impact of complete or partial thymectomy in infants undergoing cardiac surgery to treat heart congenital disorders remains a matter of hot debate [99, 100]. While systemic review of previous studies has confirmed that the procedure cause permanent changes in the T cell compartments (e.g., decreased CD4+ and CD8+ naïve T cell populations and constricted TCR repertoire) as well as T cell-related immunity, their clinical relevance in terms of increased risk of infectious diseases or malignancy has yet to be determined and needs further investigation [101]. Nevertheless, successful autotransplantation of bioengineered thymus organoids constructed from TECs isolated from the surgically removed thymus glands can help to maintain long-term T-lymphopoiesis, prevent early immunosenescence and improve $\mathrm{T}$ cell-mediated adaptive immunity. 
From a futuristic point of view, the field that will benefit most from thymus engineering will be solid organ transplantation. Despite advances in modern medicine and increased awareness of organ donation in the general population, the gap between demand and supply continues to widen rapidly over the years. In 2015 , more than 120,000 patients need a lifesaving organ transplant, and in average, 22 people die each day while on the active waiting list (http://optn.transplant.hrsa. gov). While advances in regeneration medicine and stem cell therapy can potentially fill the gap of organ shortage in the future, most of the patients are still required to undergo systemic immunosuppression throughout their life to prevent graft rejection. In addition to infection and drug-related side effects (e.g., nephrotoxicity and hypertension), patients are at much higher risk for developing a broad spectrum of cancers, compared with the general population [102]. Modulating the adaptive immune system of the recipients through thymus bioengineering might be a promising novel approach to establish immunosuppressive drug-free, donor-specific immune tolerance of allogeneic graft $[67 \bullet \bullet]$.

The idea is based on the preclinical findings that transplantation of bioengineered thymus organoids constructed with TECs co-expressing both donor- and recipient-MHCs can support the development of a $\mathrm{T}$ cell repertoire that is tolerant to both donor and recipient cells [67••]. Thymus gland will be harvested together with the needed organ from the cadaver donor and dissociated into single cell suspension via collagenase digestion. TECs and other thymic mesenchymal cells will be enriched by FACS and used to populate the decellularized scaffolds, which can be prepared from surrogate thymus glands of allogeneic or even xenogeneic origins, as components of ECM are highly conserved evolutionarily [103] and normally not antigenic (Fig. 1). The bioengineered thymus organoids will be cultured for TEC colonization and expansion ex vivo. In addition, gene expression cassettes expressing the most relevant recipient's MHC molecules will be introduced to the donor TECs via lentiviral particle transduction [104]. Meanwhile, the grafts (e.g., kidney or pancreatic islets) will be transplanted into recipients preconditioned with $\mathrm{T}$ cell depletion immunosuppressive regimen [e.g., anti-thymocyte immunoglobulin (ATG) or antiCD52 monoclonal antibody (CAMPATH)]. The bioengineered thymus organoids can be engrafted into the thoracic cavity, either by directly connecting to the internal thoracic artery to replace the endogenous thymus gland in aged recipient, which should have already shrunk and degenerated significantly, or as addition to the existing thymus in younger recipient. Once engrafted, the bioengineered thymus organoids will facilitate the generation of a new repertoire of naïve $T$ cells that can exert

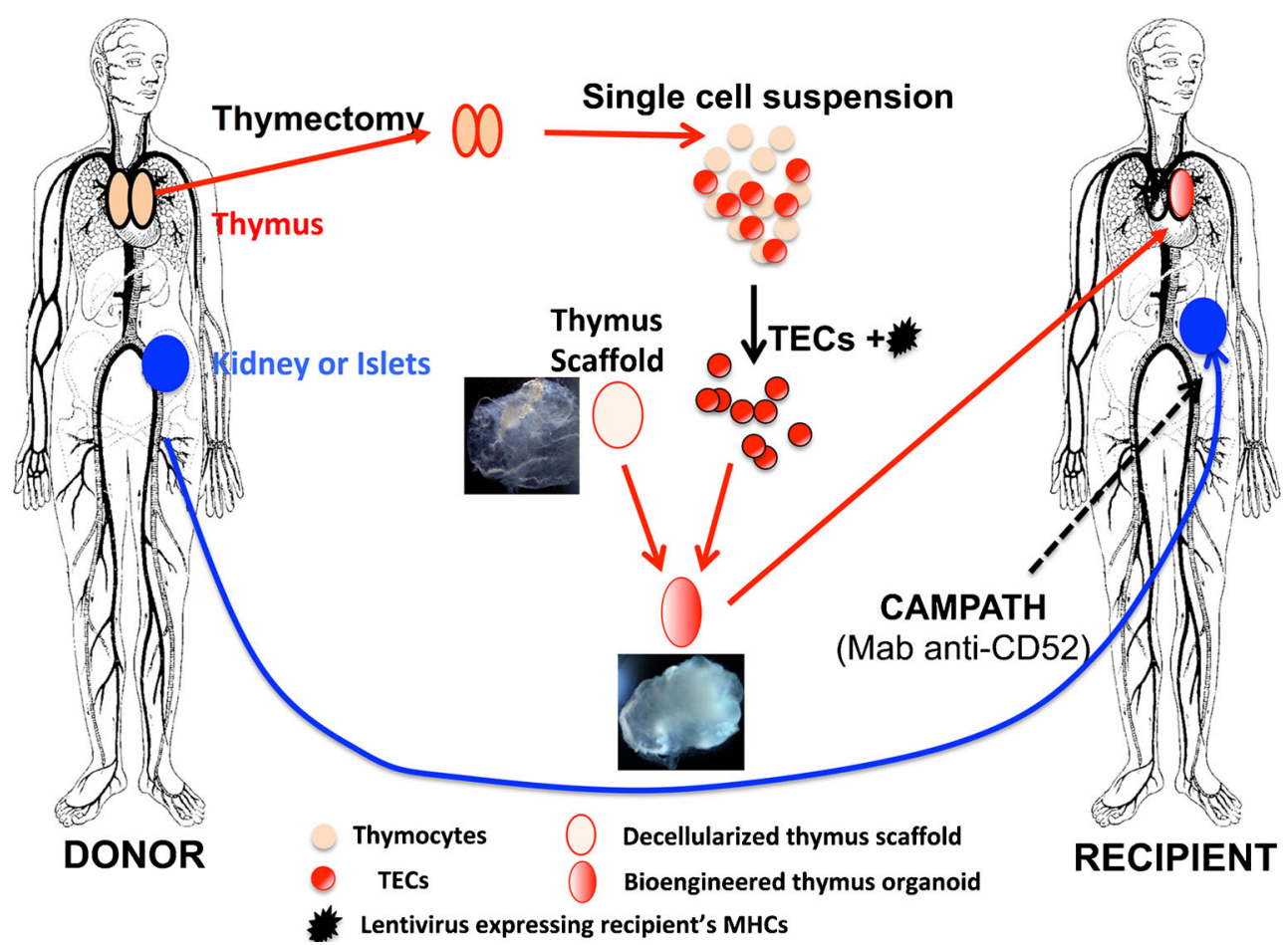

Fig. 1 Induction of donor-specific allograft tolerance by thymus bioengineering. Thymus gland is harvested from a cadaver donor in conjunction with the organ to be transplanted (e.g., kidney or pancreatic islets). Recipient, preconditioned with lymphocyte-depletion regimen [e.g., Campath (anti-CD52) or ATG (anti-thymocyte immunoglobulin)], will receive the transplant. Donor thymic epithelial cells (TECs) will be isolated from the dissociated thymus, transduced with lentiviral particles expressing the recipient's MHC molecules, and used to reconstruct the thymus organoids with surrogate thymus scaffolds. The bioengineered thymus organoid will be transplanted into the recipient, either as an additional thymus lobe (in young patients) or as a replacement (in older patients) at the time of organ transplantation. Representative images of a decellularized thymus scaffold (mouse) and a bioengineered thymus organoid (mouse) are shown 
adaptive immune function but remain unresponsiveness to donor cells, to ensure long-term survival of the transplant without the need of immunosuppression.

Another area that can potentially benefit enormously from the thymus bioengineering is to reverse immunosenescence in aged individuals. Thanks to the advances of medical sciences and modern technology, life expectancy in developed countries has increased dramatically in the past century. It is projected that by the year 2030, more than $20 \%$ of US population will be aged 65 or older. Aging is associated with progressive deterioration of immune function that leads to increased susceptibility to opportunistic infections, decreased responsiveness to vaccines, and increased incidence of autoimmunity and cancer $[105,106]$. How to prevent/reverse immunosenescence in aged individuals to ensure a healthy, quality life and to cut down societal health care-related cost remains a major challenge to medical research $[107,108]$.

Mechanistically, many of these age-related adverse events are attributed to changes in the $\mathrm{T}$ cell compartment, including a decrease in naïve $\mathrm{T}$ cells, expansion of small subsets of memory cells, and a global shift from naïve to memory phenotype. In addition, aged T cells display a number of functional defects, such as reduced proliferation, altered tonic signaling upon TCR-engagement, and reduced IL-2 production [18, 109]. Central to these age-related dysfunctions in the $\mathrm{T}$ cell compartment is age-related thymus involution [19]. Transplantation of artificial thymus organoids engineered with host MHC-expressing TEPCs derived either from multipotent stem cells (e.g., iPSCs, genetically modified hESCs) or from Foxn1-overexpressing transdifferentiated skin fibroblasts into elders can be used as an effective way to rejuvenate $\mathrm{T}$ cell immunity and treat age-related immune disorders.

\section{Potential Health Care Cost-Savings of Thymus Organoid Transplantation}

Health care spending in the USA has increased dramatically over the past 50 years, reaching 3.0 trillion dollars in 2014 that is accounted for $17.5 \%$ of the US economy. The major challenge to medical professionals and researchers is to improve the quality of medical care while decreasing its costs. While still in its infancy, recent advancements in the biomedical engineering field have demonstrated its potential not just providing novel strategies and devices to fight life-threatening diseases but also actually decreasing the cost of patient treatment while improving its outcome. For example, in patients with coronary artery disease (CAD) who undergo percutaneous coronary intervention (PCI), bioresorbable vascular scaffold (BVS) has shown short-term cost-savings and the potential to improve long-term clinical outcomes, compared with the traditional metallic drug-eluting stents [110, 111].
While the cost for thymus reconstruction and transplantation is not known at present, we estimated that its total cost is around $\$ 25,000$ to $\$ 30,000$, based on our experience with human islet isolation for treatment of patients with severe pancreatitis [112, 113]. Notably, this is similar to the annual cost of immunosuppressive drugs for patients with solid organ transplantation (e.g., liver or kidney), but the procedure will be needed only once. If, as previously discussed, donor-specific immune tolerance might be achieved by co-transplantation of the bioengineered thymus organoids, the recipients will need minimum immunosuppression, resulting in substantial healthcare savings and significant relief of patients' financial burden. For example, Medicare coverage of immunosuppressive drugs for recipients less than 65 years of age lasts only 3 years after kidney transplantation. This leaves many recipients unable to afford the necessary medication, leading to allograft rejection and premature graft failure, and the patients go back to the costly dialysis treatment, with an annual cost of more than $\$ 74,000$ [114]. With more than 15,000 patients undergo kidney transplantation each year, hundreds of millions of dollars can be saved annually if the usage of immunosuppressive drugs can be substantially reduced in this group alone.

Recently, Habka et al. performed a prospective financial analysis of transplanting bioengineered autologous liver grafts constructed with hepatocytes derived from iPSCs [115]. They estimated that constructing a liver graft with $1 \times 10^{11}$ hepatocytes (about $35 \%$ of the normal liver size) would cost 9.7 million US dollars, a number too high for clinical applications. On the contrary, a human thymus gland at puberty contains about $1-2 \times 10^{8}$ TECs [116]. If the costs for generating TECs from autologous iPSCs are similar to those of hepatocytes, the estimated cost for bioengineering a fully functional thymus organoid will be around 10,000-20,000 dollars, which is more clinically applicable. Thus, transplanting bioengineered thymus organoids might be an effective way to decrease the healthcare cost in treatment of various medical conditions, such as decreased immune protection in the elders and allograft rejection.

\section{Conclusion}

While thymus bioengineering is still at its infancy and more research is needed to further advance the technology for clinical application, preclinical studies have clearly demonstrated the proof-of-principle that it is an effective approach to rejuvenate the function of the adaptive immune system. Recent advances in stem cell research and regenerative medicine make it possible to repair and/or regenerate various tissues and/or organs in human bodies in the foreseeable future. However, immune incompatibility remains as one of the major obstacles to render the artificial organoids as integral parts 
of the hosts. Thymus bioengineering is a promising approach to modulate the adaptive immunity of the patient and achieve immunosuppression-free tissue/organ replacement.

Acknowledgments This work was supported by NIH 1R01 AI123392 (Y.F.) and the Department of Defense grant W81XWH-10-1-1055 (M.T.).

\section{Compliance with Ethical Standards}

Conflict of Interest Asako Tajima, Isha Pradhan, Massimo Trucco, and Yong Fan declare that they have no conflict of interest.

Human and Animal Rights and Informed Consent This article does not contain any studies with human or animal subjects performed by any of the authors.

\section{References}

Papers of particular interest, published recently, have been highlighted as:

- Of importance

•. Of major importance

1. Boehm T. Thymus development and function. Curr Opin Immunol. 2008;20(2):178-84. doi:10.1016/j.coi.2008.03.001.

2. Manley NR, Richie ER, Blackburn CC, Condie BG, Sage J. Structure and function of the thymic microenvironment. Front Biosci. 2012;17:2461-77.

3. Lind EF, Prockop SE, Porritt HE, Petrie HT. Mapping precursor movement through the postnatal thymus reveals specific microenvironments supporting defined stages of early lymphoid development. J Exp Med. 2001;194(2):127-34.

4. Starr TK, Jameson SC, Hogquist KA. Positive and negative selection of T cells. Annu Rev Immunol. 2003;21:139-76. doi:10. 1146/annurev.immunol.21.120601.141107.

5. Klein L, Kyewski B, Allen PM, Hogquist KA. Positive and negative selection of the $\mathrm{T}$ cell repertoire: what thymocytes see (and don't see). Nat Rev Immunol. 2014;14(6):377-91. doi:10.1038/ nri3667.

6. Kyewski B, Klein L. A central role for central tolerance. Annu Rev Immunol. 2006;24(1):571-606. doi:10.1146/annurev. immunol.23.021704.115601.

7.• Takaba H, Morishita Y, Tomofuji Y, Danks L, Nitta T, Komatsu N, et al. Fezf2 orchestrates a thymic program of self-antigen expression for immune tolerance. Cell. 2015;163(4):975-87. doi:10. 1016/j.cell.2015.10.013. This study identified a transcription factor that regulates the thymic expression of a subset of tissue restricted autoantigens that is complementary to those controlled by Aire.

8. Nagamine K, Peterson P, Scott HS, Kudoh J, Minoshima S, Heino $\mathrm{M}$, et al. Positional cloning of the APECED gene. Nat Genet. 1997;17(4):393-8. doi:10.1038/ng1297-393.

9. Anderson MS, Venanzi ES, Klein L, Chen Z, Berzins SP, Turley SJ, et al. Projection of an immunological self shadow within the thymus by the aire protein. Science. 2002;298(5597):1395-401. doi:10.1126/science.1075958.

10. Fan Y, Rudert WA, Grupillo M, He J, Sisino G, Trucco M. Thymus-specific deletion of insulin induces autoimmune diabetes. EMBO J. 2009;28(18):2812-24. doi:10.1038/emboj. 2009.212.

11. Grupillo M, Gualtierotti G, He J, Sisino G, Bottino R, Rudert WA, et al. Essential roles of insulin expression in Aire+ tolerogenic dendritic cells in maintaining peripheral self-tolerance of islet beta-cells. Cell Immunol. 2012;273(2):115-23. doi:10.1016/j. cellimm.2011.12.010.

12. Pietropaolo M, Castano L, Babu S, Buelow R, Kuo YL, Martin S, et al. Islet cell autoantigen $69 \mathrm{kD}$ (ICA69). Molecular cloning and characterization of a novel diabetes-associated autoantigen. J Clin Invest. 1993;92(1):359-71. doi:10.1172/JCI116574.

13. Fan Y, Gualtierotti G, Tajima A, Grupillo M, Coppola A, He J, et al. Compromised central tolerance of ICA69 induces multiple organ autoimmunity. J Autoimmun. 2014. doi:10.1016/j.jaut.2014. 07.001.

14. Chinn IK, Blackburn CC, Manley NR, Sempowski GD. Changes in primary lymphoid organs with aging. Semin Immunol. 2012;24(5):309-20. doi:10.1016/j.smim.2012.04.005.

15. Hale JS, Boursalian TE, Turk GL, Fink PJ. Thymic output in aged mice. Proc Natl Acad Sci U S A. 2006;103(22):8447-52. doi:10. 1073/pnas.0601040103.

16. Bertho JM, Demarquay C, Moulian N, Van Der Meeren A, BerrihAknin S, Gourmelon P. Phenotypic and immunohistological analyses of the human adult thymus: evidence for an active thymus during adult life. Cell Immunol. 1997;179(1):30-40. doi:10.1006/ cimm.1997.1148.

17. Douek DC, McFarland RD, Keiser PH, Gage EA, Massey JM, Haynes BF, et al. Changes in thymic function with age and during the treatment of HIV infection. Nature. 1998;396(6712):690-5. doi:10.1038/25374.

18. Goronzy JJ, Fang F, Cavanagh MM, Qi Q, Weyand CM. Naive T cell maintenance and function in human aging. J Immunol. 2015;194(9):4073-80. doi:10.4049/jimmunol.1500046.

19. Palmer DB. The effect of age on thymic function. Front Immunol. 2013;4:316. doi:10.3389/fimmu.2013.00316.

20. Savino W. The thymus is a common target organ in infectious diseases. PLoS Pathog. 2006;2(6):e62. doi:10.1371/journal.ppat. 0020062.

21. Ye P, Kirschner DE, Kourtis AP. The thymus during HIV disease: role in pathogenesis and in immune recovery. Curr HIV Res. 2004;2(2):177-83.

22. Lafon M, Scott-Algara D, Marche PN, Cazenave PA, JouvinMarche E. Neonatal deletion and selective expansion of mouse $\mathrm{T}$ cells by exposure to rabies virus nucleocapsid superantigen. $\mathbf{J}$ Exp Med. 1994;180(4):1207-15.

23. Auwaerter PG, Kaneshima H, McCune JM, Wiegand G, Griffin DE. Measles virus infection of thymic epithelium in the SCID-hu mouse leads to thymocyte apoptosis. J Virol. 1996;70(6):3734-40.

24. Okamoto Y, Vricella LA, Moss WJ, Griffin DE. Immature CD4+ CD8+ thymocytes are preferentially infected by measles virus in human thymic organ cultures. PLoS One. 2012;7(9):e45999. doi: 10.1371/journal.pone.0045999.

25. Sempowski GD, Hale LP, Sundy JS, Massey JM, Koup RA, Douek DC, et al. Leukemia inhibitory factor, oncostatin M, IL6 , and stem cell factor mRNA expression in human thymus increases with age and is associated with thymic atrophy. J Immunol. 2000;164(4):2180-7.

26. Herold MJ, McPherson KG, Reichardt HM. Glucocorticoids in T cell apoptosis and function. Cell Mol Life Sci. 2006;63(1):60-72. doi:10.1007/s00018-005-5390-y.

27. Bolotin E, Smogorzewska M, Smith S, Widmer M, Weinberg K. Enhancement of thymopoiesis after bone marrow transplant by in vivo interleukin-7. Blood. 1996;88(5):1887-94.

28. Berent-Maoz B, Montecino-Rodriguez E, Signer RA, Dorshkind K. Fibroblast growth factor-7 partially reverses murine thymocyte 
progenitor aging by repression of Ink4a. Blood. 2012;119(24): 5715-21. doi:10.1182/blood-2011-12-400002.

29. Danzl NM, Donlin LT, Alexandropoulos K. Regulation of medullary thymic epithelial cell differentiation and function by the signaling protein Sin. J Exp Med. 2010;207(5):999-1013. doi:10. 1084/jem.20092384.

30. Dudakov JA, Hanash AM, Jenq RR, Young LF, Ghosh A, Singer $\mathrm{NV}$, et al. Interleukin-22 drives endogenous thymic regeneration in mice. Science. 2012;336(6077):91-5. doi:10.1126/science. 1218004.

31. Taub DD, Longo DL. Insights into thymic aging and regeneration. Immunol Rev. 2005;205:72-93. doi:10.1111/j.0105-2896.2005. 00275.x.

32. Dixit VD, Yang H, Sun Y, Weeraratna AT, Youm YH, Smith RG, et al. Ghrelin promotes thymopoiesis during aging. J Clin Invest. 2007;117(10):2778-90. doi:10.1172/JCI30248.

33. van Ewijk W, Wang B, Hollander G, Kawamoto H, Spanopoulou E, Itoi $\mathrm{M}$, et al. Thymic microenvironments, 3-D versus 2-D? Semin Immunol. 1999;11(1):57-64. doi:10.1006/smim.1998.0158.

34. Nunes-Cabaco H, Sousa AE. Repairing thymic function. Curr Opin Organ Transplant. 2013;18(3):363-8. doi:10.1097/MOT, 0b013e3283615df9.

35. Corbeaux T, Hess I, Swann JB, Kanzler B, Haas-Assenbaum A, Boehm T. Thymopoiesis in mice depends on a Foxn1-positive thymic epithelial cell lineage. Proc Natl Acad Sci U S A. 2010;107(38):16613-8. doi:10.1073/pnas.1004623107.

36. Flomerfelt FA, El Kassar N, Gurunathan C, Chua KS, League SC, Schmitz S, et al. Tbata modulates thymic stromal cell proliferation and thymus function. J Exp Med. 2010;207(11):2521-32. doi:10. 1084/jem.20092759.

37. Saade M, Irla M, Yammine M, Boulanger N, Victorero G, Vincentelli R, et al. Spatial (Tbata) expression in mature medullary thymic epithelial cells. Eur J Immunol. 2010;40(2):530-8. doi:10.1002/eji.200939605.

38. Nowell CS, Bredenkamp N, Tetelin S, Jin X, Tischner C, Vaidya $\mathrm{H}$, et al. Foxn1 regulates lineage progression in cortical and medullary thymic epithelial cells but is dispensable for medullary sublineage divergence. Plos Genet. 2011;7(11):e1002348. doi: 10.1371/journal.pgen.1002348.

39. Masuda K, Germeraad WT, Satoh R, Itoi M, Ikawa T, Minato N, et al. Notch activation in thymic epithelial cells induces development of thymic microenvironments. Mol Immunol. 2009;46(8-9): 1756-67. doi:10.1016/j.molimm.2009.01.015.

40. Sun TT, Bonitz P, Burns WH. Cell culture of mammalian thymic epithelial cells: growth, structural, and antigenic properties. Cell Immunol. 1984;83(1):1-13.

41. Saunders DJ, Georgiou HM, Wu L, Shortman K. Induction of limited growth and differentiation of early thymic precursor cells by thymic epithelial cell lines. Immunol Lett. 1995;47(1-2):45-51.

42. Bonfanti P, Claudinot S, Amici AW, Farley A, Blackburn CC, Barrandon Y. Microenvironmental reprogramming of thymic epithelial cells to skin multipotent stem cells. Nature. 2010;466(7309):978-82. doi:10.1038/nature09269.

43. Anderson G, Jenkinson EJ. Fetal thymus organ culture. CSH Protoc. 2007. doi:10.1101/pdb.prot4808.

44. Marshall D, Bagley J, Le P, Hogquist K, Cyr S, Von Schild E, et al. $T$ cell generation including positive and negative selection ex vivo in a three-dimensional matrix. J Hematother Stem Cell Res. 2003;12(5):565-74. doi:10.1089/152581603322448277.

45. Chung B, Montel-Hagen A, Ge S, Blumberg G, Kim K, Klein S, et al. Engineering the human thymic microenvironment to support thymopoiesis in vivo. Stem Cells. 2014;32(9):2386-96. doi:10. 1002/stem.1731. This study shows that human thymic mesenchyme genetically engineered to express VEGF can support human thymic epithelial cell culture in vitro and promote T-cell development in vivo.
46. Seach N, Mattesich M, Abberton K, Matsuda K, Tilkorn DJ, Rophael J, et al. Vascularized tissue engineering mouse chamber model supports thymopoiesis of ectopic thymus tissue grafts. Tissue Eng Part C Methods. 2010;16(3):543-51. doi:10.1089/ ten.TEC.2009.0135.

47. Poznansky MC, Evans RH, Foxall RB, Olszak IT, Piascik AH, Hartman KE, et al. Efficient generation of human T cells from a tissue-engineered thymic organoid. Nat Biotechnol. 2000;18(7): 729-34. doi:10.1038/77288.

48. Pinto S, Schmidt K, Egle S, Stark HJ, Boukamp P, Kyewski B. An organotypic coculture model supporting proliferation and differentiation of medullary thymic epithelial cells and promiscuous gene expression. J Immunol. 2013;190(3):1085-93. doi:10.4049/ jimmunol.1201843.

49. Buck CA, Horwitz AF. Cell surface receptors for extracellular matrix molecules. Annu Rev Cell Biol. 1987;3:179-205. doi:10. 1146/annurev.cb.03.110187.001143.

50. Mammoto T, Mammoto A, Ingber DE. Mechanobiology and developmental control. Annu Rev Cell Dev Biol. 2013;29:27-61. doi:10.1146/annurev-cellbio-101512-122340.

51. Watt FM, Huck WT. Role of the extracellular matrix in regulating stem cell fate. Nat Rev Mol Cell Biol. 2013;14(8):467-73. doi:10. 1038/nrm3620.

52. Lim CJ, Kain KH, Tkachenko E, Goldfinger LE, Gutierrez E, Allen MD, et al. Integrin-mediated protein kinase A activation at the leading edge of migrating cells. Mol Biol Cell. 2008;19(11): 4930-41. doi:10.1091/mbc.E08-06-0564.

53. Daley WP, Yamada KM. ECM-modulated cellular dynamics as a driving force for tissue morphogenesis. Curr Opin Genes Dev. 2013;23(4):408-14. doi:10.1016/j.gde.2013.05.005.

54. Bajaj P, Schweller RM, Khademhosseini A, West JL, Bashir R. 3D biofabrication strategies for tissue engineering and regenerative medicine. Annu Rev Biomed Eng. 2014;16:247-76. doi:10. 1146/annurev-bioeng-071813-105155.

55. Gilbert TW, Sellaro TL, Badylak SF. Decellularization of tissues and organs. Biomaterials. 2006;27(19):3675-83. doi:10.1016/j. biomaterials.2006.02.014

56. Baptista PM, Orlando G, Mirmalek-Sani SH, Siddiqui M, Atala A, Soker S. Whole organ decellularization - a tool for bioscaffold fabrication and organ bioengineering. Conf Proc IEEE Eng Med Biol Soc. 2009;2009:6526-9. doi:10.1109/IEMBS.2009. 5333145 .

57. Orlando G, Soker S, Stratta RJ. Organ bioengineering and regeneration as the new Holy Grail for organ transplantation. Ann Surg. 2013;258(2):221-32. doi:10.1097/SLA.0b013e31829c79cf.

58. Booth C, Soker T, Baptista P, Ross CL, Soker S, Farooq U, et al. Liver bioengineering: current status and future perspectives. World J Gastroenterol. 2012;18(47):6926-34. doi:10.3748/wjg. v18.i47.6926.

59. Orlando G, Baptista P, Birchall M, De Coppi P, Farney A, Guimaraes-Souza NK, et al. Regenerative medicine as applied to solid organ transplantation: current status and future challenges. Transpl Int. 2011;24(3):223-32. doi:10.1111/j.1432-2277.2010. 01182.x.

60. Ott HC, Matthiesen TS, Goh SK, Black LD, Kren SM, Netoff TI, et al. Perfusion-decellularized matrix: using nature's platform to engineer a bioartificial heart. Nat Med. 2008;14(2):213-21. doi: $10.1038 / \mathrm{nm} 1684$.

61. Ott HC, Clippinger B, Conrad C, Schuetz C, Pomerantseva I, Ikonomou L, et al. Regeneration and orthotopic transplantation of a bioartificial lung. Nat Med. 2010;16(8):927-33. doi:10. 1038/nm.2193.

62. Petersen TH, Calle EA, Zhao L, Lee EJ, Gui L, Raredon MB, et al. Tissue-engineered lungs for in vivo implantation. Science. 2010;329(5991):538-41. doi:10.1126/science.1189345. 
63. Uygun BE, Soto-Gutierrez A, Yagi H, Izamis ML, Guzzardi MA, Shulman C, et al. Organ reengineering through development of a transplantable recellularized liver graft using decellularized liver matrix. Nat Med. 2010;16(7):814-20. doi:10.1038/nm.2170.

64. Goh SK, Bertera S, Olsen P, Candiello JE, Halfter W, Uechi G, et al. Perfusion-decellularized pancreas as a natural 3D scaffold for pancreatic tissue and whole organ engineering. Biomaterials. 2013;34(28):6760-72. doi:10.1016/j.biomaterials.2013.05.066.

65. Macchiarini P, Jungebluth P, Go T, Asnaghi MA, Rees LE, Cogan TA, et al. Clinical transplantation of a tissue-engineered airway. Lancet. 2008;372(9655):2023-30. doi:10.1016/S0140-6736(08) 61598-6.

66. Fedak PW, de Sa MP, Verma S, Nili N, Kazemian P, Butany J, et al. Vascular matrix remodeling in patients with bicuspid aortic valve malformations: implications for aortic dilatation. J Thorac Cardiovasc Surg. 2003;126(3):797-806.

67.• Fan Y, Tajima A, Goh SK, Geng X, Gualtierotti G, Grupillo M, et al. Bioengineering thymus organoids to restore thymic function and induce donor-specific immune tolerance to allografts. Mol Ther. 2015. doi:10.1038/mt.2015.77. This study shows that functional thymus organoids can be generated by repopulating decellularized 3-D thymic scaffolds with isolated thymic epithelial cells (TECs) and that introducing allogeneic TECs in the bioengieered thymus organoids can induce donor specific immune tolerance.

68. Tajima A, Liu W, Pradhan I, Bertera S, Bagia C, Trucco M, et al. Bioengineering mini functional thymic units with EAK16-II/ EAKIIH6 self-assembling hydrogel. Clin Immunol. 2015. doi: 10.1016/j.clim.2015.03.010.

69. Orlando G, Soker S, Stratta RJ, Atala A. Will regenerative medicine replace transplantation? Cold Spring Harb Perspect Med. 2013;3(8):a015693. doi:10.1101/cshperspect.a015693.

70. Cuddihy AR, Ge S, Zhu J, Jang J, Chidgey A, Thurston G, et al. VEGF-mediated cross-talk within the neonatal murine thymus. Blood. 2009;113(12):2723-31. doi:10.1182/blood-2008-06162040.

71. Cuddihy AR, Suterwala BT, Ge S, Kohn LA, Jang J, Andrade J, et al. Rapid thymic reconstitution following bone marrow transplantation in neonatal mice is VEGF-dependent. Biol Blood Marrow Transplant. 2012;18(5):683-9. doi:10.1016/j.bbmt.2012.01.006.

72. Okabe M, Ito S, Nishio N, Tanaka Y, Isobe K. Thymic epithelial cells induced from pluripotent stem cells by a three-dimensional spheroid culture system regenerates functional $\mathrm{T}$ cells in nude mice. Cell Reprogram. 2015;17(5):368-75. doi:10.1089/cell. 2015.0006.

73. Kim MJ, Miller CM, Shadrach JL, Wagers AJ, Serwold T. Young, proliferative thymic epithelial cells engraft and function in aging thymuses. J Immunol. 2015;194(10):4784-95. doi:10.4049/ jimmunol.1403158.

74. Ucar A, Ucar O, Klug P, Matt S, Brunk F, Hofmann TG, et al. Adult thymus contains FoxN1(-) epithelial stem cells that are bipotent for medullary and cortical thymic epithelial lineages. Immunity. 2014;41(2):257-69. doi:10.1016/j.immuni.2014.07. 005. This study shows the presence of bipotential TEC progenitors in adult thymus.

75. Rossi SW, Jenkinson WE, Anderson G, Jenkinson EJ. Clonal analysis reveals a common progenitor for thymic cortical and medullary epithelium. Nature. 2006;441(7096):988-91. doi:10. 1038/nature04813.

76. Depreter MG, Blair NF, Gaskell TL, Nowell CS, Davern K, Pagliocca A, et al. Identification of Plet-1 as a specific marker of early thymic epithelial progenitor cells. Proc Natl Acad Sci U S A. 2008;105(3):961-6. doi:10.1073/pnas.0711170105.

77. Bleul CC, Corbeaux T, Reuter A, Fisch P, Monting JS, Boehm T. Formation of a functional thymus initiated by a postnatal epithelial progenitor cell. Nature. 2006;441(7096):992-6. doi:10.1038/ nature 04850 .

78. Boehm T, Bleul CC. Thymus-homing precursors and the thymic microenvironment. Trends Immunol. 2006;27(10):477-84. doi: 10.1016/j.it.2006.08.004.

79. Blanpain C, Fuchs E. Stem cell plasticity. Plasticity of epithelial stem cells in tissue regeneration. Science. 2014;344(6189): 1242281. doi:10.1126/science. 1242281.

80. Hamazaki Y. Adult thymic epithelial cell (TEC) progenitors and TEC stem cells: models and mechanisms for TEC development and maintenance. Eur J Immunol. 2015. doi:10.1002/eji. 201545844.

81. Dumont-Lagace M, Brochu S, St-Pierre C, Perreault C. Adult thymic epithelium contains nonsenescent label-retaining cells. J Immunol. 2014;192(5):2219-26. doi:10.4049/jimmunol. 1302961. This study shows the presence of a population of putative TEC progenitors in adult thymus that can remain quiescent and are protected from sensescence.

82. Osada M, Singh VJ, Wu K, Sant'Angelo DB, Pezzano M. Label retention identifies a multipotent mesenchymal stem cell-like population in the postnatal thymus. PLoS One. 2013;8(12):e83024. doi:10.1371/journal.pone.0083024.

83. Gray DH, Seach N, Ueno T, Milton MK, Liston A, Lew AM, et al. Developmental kinetics, turnover, and stimulatory capacity of thymic epithelial cells. Blood. 2006;108(12):3777-85. doi:10.1182/ blood-2006-02-004531.

84. Wong K, Lister NL, Barsanti M, Lim JM, Hammett MV, Khong DM, et al. Multilineage potential and self-renewal define an epithelial progenitor cell population in the adult thymus. Cell Rep. 2014;8(4):1198-209. doi:10.1016/j.celrep.2014.07.029. This study shows the presence of TEC progenitors in adult thymus.

85. Boehm T, Swann JB. Thymus involution and regeneration: two sides of the same coin? Nat Rev Immunol. 2013;13(11):831-8. doi:10.1038/nri3534.

86. Klug DB, Crouch E, Carter C, Coghlan L, Conti CJ, Richie ER. Transgenic expression of cyclin D1 in thymic epithelial precursors promotes epithelial and $\mathrm{T}$ cell development. J Immunol. 2000;164(4):1881-8.

87. Senoo M, Pinto F, Crum CP, McKeon F. p63 Is essential for the proliferative potential of stem cells in stratified epithelia. Cell. 2007;129(3):523-36. doi:10.1016/j.cell.2007.02.045.

88. Candi E, Rufini A, Terrinoni A, Giamboi-Miraglia A, Lena AM, Mantovani R, et al. DeltaNp63 regulates thymic development through enhanced expression of FgfR2 and Jag2. Proc Natl Acad Sci U S A. 2007;104(29):11999-2004. doi:10.1073/pnas. 0703458104.

89. Rode I, Martins VC, Kublbeck G, Maltry N, Tessmer C, Rodewald HR. Foxn1 protein expression in the developing, aging, and regenerating thymus. J Immunol. 2015. doi:10.4049/ jimmunol.1502010.

90. Chen L, Xiao S, Manley NR. Foxn1 is required to maintain the postnatal thymic microenvironment in a dosage-sensitive manner. Blood. 2009;113(3):567-74. doi:10.1182/blood-2008-05-156265.

91. Parent AV, Russ HA, Khan IS, LaFlam TN, Metzger TC, Anderson MS, et al. Generation of functional thymic epithelium from human embryonic stem cells that supports host T cell development. Cell Stem Cell. 2013;13(2):219-29. doi:10.1016/j.stem. 2013.04.004. This study, together with the study of Sun et al., demonstrates that human embryonic stem cells can be induced to differentiate into thymic epithelial progenitor-like cells.

92. Sun X, Xu J, Lu H, Liu W, Miao Z, Sui X, et al. Directed differentiation of human embryonic stem cells into thymic epithelial progenitor-like cells reconstitutes the thymic microenvironment in vivo. Cell Stem Cell. 2013;13(2):230-6. doi:10.1016/j.stem. 2013.06.014. This study, together with the study of Parent 
et al., demonstrates that human embryonic stem cells can be induced to differentiate into thymic epithelial progenitor-like cells.

93. Lai L, Jin J. Generation of thymic epithelial cell progenitors by mouse embryonic stem cells. Stem Cells. 2009;27(12):3012-20. doi:10.1002/stem.238.

94. Soh CL, Giudice A, Jenny RA, Elliott DA, Hatzistavrou T, Micallef SJ, et al. FOXN1 (GFP/w) reporter hESCs enable identification of integrin-beta4, HLA-DR, and EpCAM as markers of human PSC-derived FOXN1(+) thymic epithelial progenitors. Stem Cell Rep. 2014;2(6):925-37. doi:10.1016/j.stemcr.2014. 04.009.

95. Inami Y, Yoshikai T, Ito S, Nishio N, Suzuki H, Sakurai H, et al. Differentiation of induced pluripotent stem cells to thymic epithelial cells by phenotype. Immunol Cell Biol. 2011;89(2):314-21. doi:10.1038/icb.2010.96.

96.• Bredenkamp N, Ulyanchenko S, O’Neill KE, Manley NR, Vaidya $\mathrm{HJ}$, Blackburn CC. An organized and functional thymus generated from FOXN1-reprogrammed fibroblasts. Nat Cell Biol. 2014;16(9):902-8. doi:10.1038/ncb3023. This study shows that forced expression of Foxn1 can transdifferentiate mouse embryonic fibroblasts into thymic epithelial cell (TEC)-like cells that can support T-cell development, highlighting an alternative source of TECs for thymus bioengineering.

97. Bredenkamp N, Nowell CS, Blackburn CC. Regeneration of the aged thymus by a single transcription factor. Development. 2014;141(8):1627-37. doi:10.1242/dev.103614.

98. Albuquerque AS, Marques JG, Silva SL, Ligeiro D, Devlin BH, Dutrieux J, et al. Human FOXN1-deficiency is associated with alphabeta double-negative and FoxP3 + T-cell expansions that are distinctly modulated upon thymic transplantation. PLoS One. 2012;7(5):e37042. doi:10.1371/journal.pone.0037042.

99. Roosen J, Oosterlinck W, Meyns B. Routine thymectomy in congenital cardiac surgery changes adaptive immunity without clinical relevance. Interact Cardiovasc Thorac Surg. 2015;20(1):1016. doi:10.1093/icvts/ivu343.

100. Turan T, Turan A, Arslan C, Kinoglu B, Sarioglu T. How does neonatal thymectomy affect the immune system? Acta Cardiol. 2004;59(5):511-3

101. Afifi A, Raja SG, Pennington DJ, Tsang VT. For neonates undergoing cardiac surgery does thymectomy as opposed to thymic preservation have any adverse immunological consequences? Interact Cardiovasc Thorac Surg. 2010;11(3):287-91. doi:10. 1510/icvts.2010.237172.

102. Engels EA, Pfeiffer RM, Fraumeni Jr JF, Kasiske BL, Israni AK, Snyder JJ, et al. Spectrum of cancer risk among US solid organ transplant recipients. JAMA. 2011;306(17):1891-901. doi:10. 1001/jama.2011.1592.

103. Bernard MP, Chu ML, Myers JC, Ramirez F, Eikenberry EF, Prockop DJ. Nucleotide sequences of complementary deoxyribonucleic acids for the pro alpha 1 chain of human type I procollagen. Statistical evaluation of structures that are conserved during evolution. Biochemistry. 1983;22(22):5213-23.

104. Escors D, Breckpot K. Lentiviral vectors in gene therapy: their current status and future potential. Arch Immunol Ther Exp (Warsz). 2010;58(2):107-19. doi:10.1007/s00005-010-0063-4.

105. Haralambieva IH, Painter SD, Kennedy RB, Ovsyannikova IG, Lambert ND, Goergen KM, et al. The impact of immunosenescence on humoral immune response variation after influenza $\mathrm{A} / \mathrm{H} 1 \mathrm{~N} 1$ vaccination in older subjects. PLoS One. 2015;10(3):e0122282. doi:10.1371/journal.pone.0122282.

106. Nikolich-Zugich J, Rudd BD. Immune memory and aging: an infinite or finite resource? Curr Opin Immunol. 2010;22(4):53540. doi:10.1016/j.coi.2010.06.011.

107. Black S, De Gregorio E, Rappuoli R. Developing vaccines for an aging population. Sci Transl Med. 2015;7(281):281ps8. doi:10. 1126/scitranslmed.aaa0722.

108. Aw D, Silva AB, Palmer DB. Immunosenescence: emerging challenges for an ageing population. Immunology. 2007;120(4):43546. doi:10.1111/j.1365-2567.2007.02555.x.

109. Ahmed M, Lanzer KG, Yager EJ, Adams PS, Johnson LL, Blackman MA. Clonal expansions and loss of receptor diversity in the naive CD8 $\mathrm{T}$ cell repertoire of aged mice. J Immunol. 2009;182(2):784-92.

110. Stone GW, Gao R, Kimura T, Kereiakes DJ, Ellis SG, Onuma Y, et al. 1-year outcomes with the absorb bioresorbable scaffold in patients with coronary artery disease: a patient-level, pooled metaanalysis. Lancet. 2016. doi:10.1016/S0140-6736(15)01039-9.

111. Muramatsu T, Onuma Y, van Geuns RJ, Chevalier B, Patel TM, Seth A, et al. 1-year clinical outcomes of diabetic patients treated with everolimus-eluting bioresorbable vascular scaffolds: a pooled analysis of the ABSORB and the SPIRIT trials. J Am Coll Cardiol Intv. 2014;7(5):482-93. doi:10.1016/j.jcin.2014.01.155.

112. Bottino R, Trucco M. Clinical implementation of islet transplantation: a current assessment. Pediatr Diabetes. 2015;16(6):393401. doi:10.1111/pedi.12287.

113. Johnston PC, Lin YK, Walsh RM, Bottino R, Stevens TK, Trucco $\mathrm{M}$, et al. Factors associated with islet yield and insulin independence after total pancreatectomy and islet cell autotransplantation in patients with chronic pancreatitis utilizing off-site islet isolation: Cleveland clinic experience. J Clin Endocrinol Metab. 2015;100(5):1765-70. doi:10.1210/jc.2014-4298.

114. Tanriover B, Stone PW, Mohan S, Cohen DJ, Gaston RS. Future of medicare immunosuppressive drug coverage for kidney transplant recipients in the United States. Clin J Am Soc Nephrol. 2013;8(7):1258-66. doi:10.2215/CJN.09440912.

115. Habka D, Mann D, Landes R, Soto-Gutierrez A. Future economics of liver transplantation: a 20-year cost modeling forecast and the prospect of bioengineering autologous liver grafts. PLoS One. 2015;10(7):e0131764. doi:10.1371/journal.pone.0131764.

116. Lynch HE, Goldberg GL, Chidgey A, Van den Brink MR, Boyd R, Sempowski GD. Thymic involution and immune reconstitution. Trends Immunol. 2009;30(7):366-73. doi:10.1016/j.it.2009.04.003. 